\title{
Transport and Magnetic Properties of $\mathrm{YbCu}_{4} \mathrm{Ni}$
}

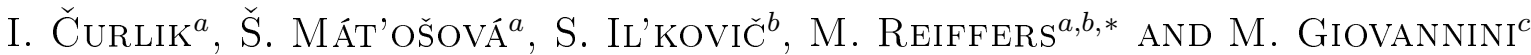 \\ ${ }^{a}$ Institute of Experimental Physics, Watsonova 47, SK 04353 Košice, Slovakia \\ ${ }^{b}$ Faculty of Sciences, University of Prešov, 17. novembra 1, SK 08078 Prešov, Slovakia \\ ${ }^{c}$ CNR-SPIN and Department of Chemistry, University of Genova, I-16146 Genova, Italy
}

\begin{abstract}
The results on the influence of magnetic field on the electrical resistivity $\rho(T)$ of the heavy fermion $\mathrm{YbCu}_{4} \mathrm{Ni}$ in the temperature range $0.4-300 \mathrm{~K}$ are presented. We have observed a Kondo-like behaviour below $10 \mathrm{~K}$ with a minimum in $\rho(T)$ at about $60 \mathrm{~K}$. An applied magnetic field depresses this behaviour, and a maximum in $\rho(T)$ appears similarly to the maximum observed in heat capacity. The observed electrical magnetoresistance shows negative values in all applied magnetic fields and with increasing temperature its absolute magnitude increases. Moreover, we extended our previous susceptibility measurements up to high temperatures of $1000 \mathrm{~K}$, in order to study possible mixed valence behaviour, which however was not observed.
\end{abstract}

PACS: 71.27.+a, 75.30.-m, 75.30.Mb

\section{Introduction}

In recent years a growing interest has been devoted to Yb-based systems due to the variety of anomalous behaviours which could occur at low temperatures $[1,2]$. In fact, a certain number of $\mathrm{Yb}$ compounds show strong correlation between electrons, due to hybridization of $f$-electrons and conduction electrons. Because of the possibility of Yb-atoms to be in a divalent or a trivalent state, a possible coexistence of both states may occur as heterogeneous mixed valence systems, or Yb-ions could have some non-integer valence as it happens in intermediate valence systems [3]. The interest in our topic was triggered by the previous investigation made on the heavy fermions $\mathrm{YbCu}_{4} \mathrm{~T}(\mathrm{~T}=\mathrm{Ag}, \mathrm{Au})$, which crystallize in an ordered derivative of the $\mathrm{AuBe}_{5}$-type (cubic $\mathrm{MgCu}_{4} \mathrm{Sn}$ type), and the parent compound is the dense Kondo system $\mathrm{YbCu}_{5}[4-6]$. It was found that $\mathrm{YbCu}_{4} \mathrm{Au}$ orders antiferromagnetically below $1 \mathrm{~K}$, whereas $\mathrm{YbCu}_{4} \mathrm{Ag}$ has a nonmagnetic ground state [7].

Recently the new compound $\mathrm{YbCu}_{4} \mathrm{Ni}$ has been studied by us [8]. It was discovered that this compound is a new heavy fermion (HF) member of the series of $\mathrm{YbCu}_{4} \mathrm{M}$ $\left(\mathrm{M}=\right.$ metal), which crystallizes in the cubic $\mathrm{MgCu}_{4} \mathrm{Sn}$ type structure. The measurements of the heat capacity revealed a possible magnetic ordering below $0.5 \mathrm{~K}$, which was supported by susceptibility measurements. However, the transport properties have not been studied yet. In order to continue the work previously done on $\mathrm{YbCu}_{4} \mathrm{Ni}$ [8], we present in this paper the results of the study of the electrical resistivity of this compound in the temperature range $0.4-300 \mathrm{~K}$, made in zero and applied magnetic fields up to $9 \mathrm{~T}$. Moreover, in order to check the existence of a possible intermediate valence behaviour, the measurements of the susceptibility was extended up

* corresponding author; e-mail: reiffers@saske.sk to $1000 \mathrm{~K}$. In fact, the existence of a maximum in the susceptibility at high temperatures would be an indication of intermediate valence phenomena as is the case of $\mathrm{YbAl}_{4} \mathrm{Ni}[9]$.

\section{Experimental}

We have used in measurements the same polycrystalline sample of $\mathrm{YbCu}_{4} \mathrm{Ni}$, which has already been characterized in our previous work. The details on preparation have been reported [8].

The AC electrical resistivity and magnetoresistivity measurements were performed by PPMS commercial device (Quantum Design) in the temperature range 0.4 $300 \mathrm{~K}$ and in applied magnetic fields up to $9 \mathrm{~T}$. The high temperature susceptibility measurements were performed by VERSALAB commercial device (Quantum Design) in Presov by VSM magnetometer in the temperature range $300-1000 \mathrm{~K}$ and in an applied magnetic field of $1000 \mathrm{Oe.}$

\section{Results and discussion}

The temperature dependence of the electrical resistivity $\rho(T)$ of $\mathrm{YbCu}_{4} \mathrm{Ni}$ taken in several magnetic fields is presented in Fig. 1. One could see that the electrical resistivity decreases with decreasing temperature. At about $60 \mathrm{~K}$ it first reaches a minimum and then it starts to increase with further decreasing temperature. Below $10 \mathrm{~K} \rho(T)$ for $B=0 \mathrm{~T}$ shows a $-\ln T$ behaviour, which is typical of a Kondo system. We have not observed however the maximum in $\rho(T)$ down to $0.4 \mathrm{~K}$, although the heat capacity shows a possible magnetic ordering below $0.5 \mathrm{~K}$. In order to clarify this point, the measurements in the $\mathrm{mK}$ range are necessary. The behaviour is similar to those of other heavy fermions as $\mathrm{YbCu}_{4} \mathrm{~T}(\mathrm{~T}=\mathrm{Ag}$, $\mathrm{Au})[7]$.

The applied magnetic field depresses such a behavior and, in agreement with the heat capacity behaviour [8], 


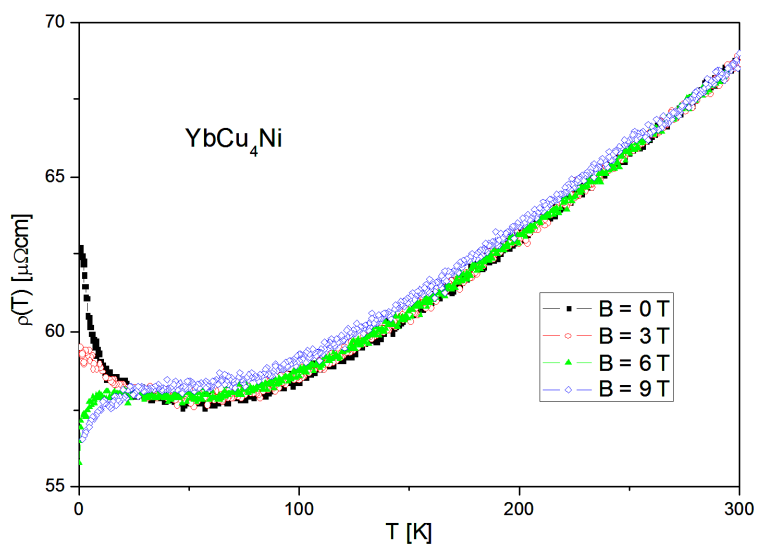

Fig. 1. Temperature dependence of the electrical resistivity $\rho(T)$ of $\mathrm{YbCu}_{4} \mathrm{Ni}$ measured in several magnetic field strength.

a maximum at low temperatures appears, as it is presented in detail in Fig. 2. This maximum occurs at the same temperature as that in heat capacity [8] (like a Schottky type maximum) and moves to higher temperatures with increasing magnetic field strength. Considering the results of our previous work [8], our data may indicate a scenario with a transition from a single impurity Kondo regime at high temperatures into a coherent Kondo lattice at low temperatures. Valence fluctuations are ruled out as it will be discussed below.

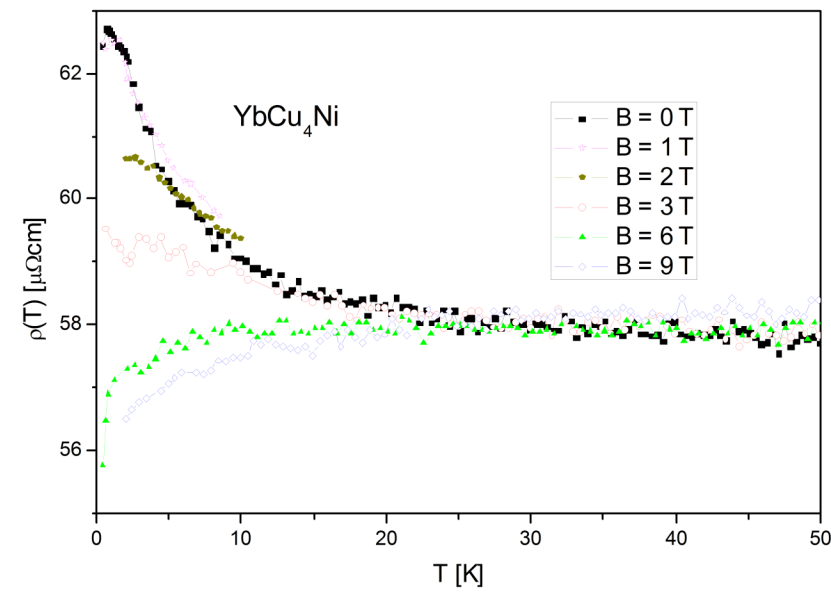

Fig. 2. Low temperature details of the temperature dependence of the electrical resistivity $\rho(T)$ of $\mathrm{YbCu}_{4} \mathrm{Ni}$ measured in several magnetic field strength.

In Fig. 3 the electrical magnetoresitivity $[(\rho(T, B)-$ $\rho(T, 0)) / \rho(T, 0)] \cdot 100(\mathrm{MR})$ of $\mathrm{YbCu}_{4} \mathrm{Ni}$ as a function of temperature is shown. Negative magnetoresistivity values in all the applied magnetic fields are observed. At the lowest temperatures the electrical magnetoresitivity reaches $10 \%$ at a magnetic field of $9 \mathrm{~T}$. It has the tendency to saturation near $9 \mathrm{~T}$. With increasing temperatures the magnitude of MR decreases.

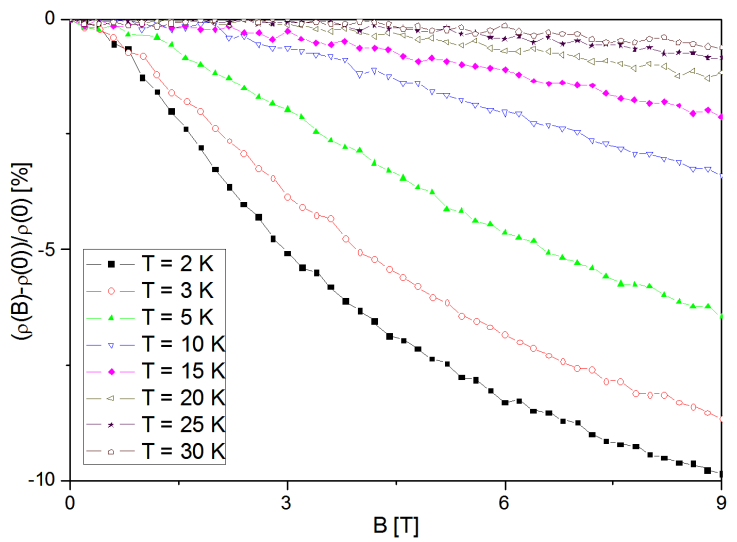

Fig. 3. Electrical magnetoresitivity $(\rho(T, B) \quad-$ $\rho(T, 0)) / \rho(T, 0) \cdot 100$ of $\mathrm{YbuCu}_{4} \mathrm{Ni}$ as a function of temperature.

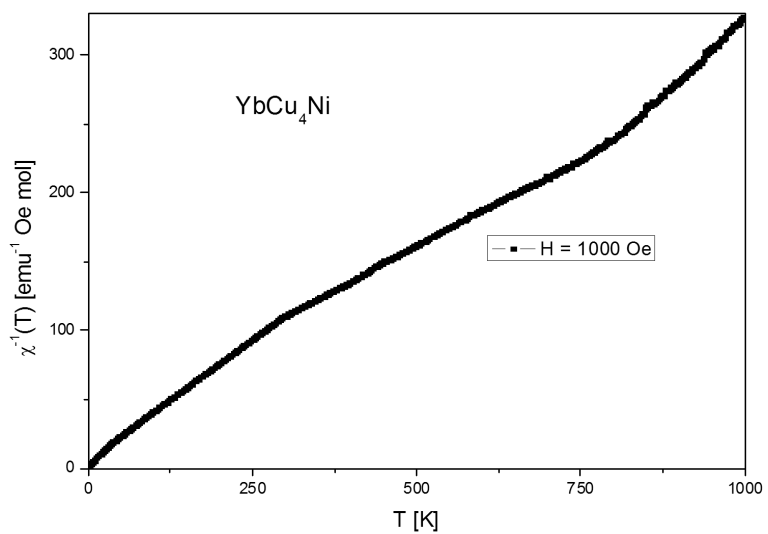

Fig. 4. Temperature dependence of the reciprocal magnetic susceptibility $\chi^{-1}(T)$ of $\mathrm{YbCu}_{4} \mathrm{Ni}$.

In order to check for any existence of intermediate valence behaviour in $\mathrm{YbCu}_{4} \mathrm{Ni}$ we have measured the susceptibility up to $1000 \mathrm{~K}$. In case of the intermediate valence systems, a maximum in the susceptibility at high temperatures is expected to appear [9]. However, as shown in Fig. 4, this is not the case of $\mathrm{YbCu}_{4} \mathrm{Ni}$.

\section{Conclusion}

In conclusion, we have studied the influence of magnetic field on the temperature dependence of electrical resistivity of the heavy fermion $\mathrm{YbCu}_{4} \mathrm{Ni}$ down to $0.4 \mathrm{~K}$. We have observed the Kondo-like behaviour below $10 \mathrm{~K}$ with a minimum in $\rho(T)$ at about $60 \mathrm{~K}$. Magnetic field depresses this behaviour and in consequence, a maximum in $\rho(T)$ appears similarly to the maximum observed in heat capacity under applied magnetic field. Electrical magnetoresistivity is negative and with increasing temperature its magnitude decreases. Moreover, we extended our previous susceptibility measurements up to high temperatures of $1000 \mathrm{~K}$, in order to check for the existence of intermediate valence behaviour, which was not observed. 


\section{Acknowledgments}

This work has been partly supported by the SlovakItalian Scientific-Technological Exchange Program for fellowships in Genova and Kosice; Slovak Research and Development Agency under the contract No. APVV SK-IT-0023-08 and VVCE-0058-07; the Slovak grant agency VEGA $2 / 0007 / 09$; the CLTP as the Centre of Excellence of the Slovak Academy of Sciences and P.J. Safárik University; the CEX Nanofluid as the Centre of Excellence SAS and by 7th FP EU - MICROKELVIN. The liquid nitrogen for the experiment has been sponsored by the U.S. Steel Kosice, s.r.o.

\section{References}

[1] M. Giovannini, E. Bauer, H. Michor, G. Hilscher, A. Galatanu, A. Saccone, P. Rogl, Intermetallics $\mathbf{9}$, 481 (2001).

[2] E. Bauer, G. Hischer, H. Michor, Ch. Paul, Y. Aoki, H. Sato, D.T. Adroja, J.-G. Park, P. Bonville, J. Sereni, M. Giovannini, A. Saccone, J. Phys. 17, S999 (2005)
[3] Proc. Int. Conf. on Valence Instabilities, Zürich, Eds. P. Wachter, H. Boppart, North-Holland, Amsterdam 1982.

[4] N. Tsujii, J. He, K. Yoshimura, K. Kosuge, H. Michor, K. Kreiner, G. Hilscher, Phys. Rev. B 55, 1032 (1997).

[5] M. Giovannini, A. Saccone, St. Muller, H. Michor, E. Bauer, J. Phys, Condens. Matter 17, S877 (2005).

[6] M. Giovannini, R. Pasero, S. De Negri, A. Saccone, Intermetallics 16, 399 (2008).

[7] C. Rossel, K.N. Yang, M.B. Maple, Z. Fisk, E. Zirngiebl, J.D. Thompson, Phys. Rev. B 35, 1914 (1987).

[8] I. Čurlik, M. Reiffers, M. Giovannini, E. Gažo, J. Šebek, E. Santavá, Acta Phys. Pol. A 118, 919 (2010).

[9] A. Kowalczyk, M. Falkowski, T. Tolinski, J. Appl. Phys. 107, 123917 (2010). 